\title{
Appendix: An Evolution-based Approach for Efficient Differentiable Architecture Search
}

\author{
Masayuki Kobayashi \\ Yokohama National University \\ Yokohama, Japan \\ kobayashi-masayuki-xc@ynu.jp
}

\author{
Tomoharu Nagao \\ Yokohama National University \\ Yokohama, Japan \\ nagao@ynu.ac.jp
}

\section{SEARCH SPACE}

We followed the search space provided in $[8,9,11,15]$, in which multiple cells are stacked together to form a deep neural network. Our search space $O$ included the following operations: identity, $3 \times 3$ and $5 \times 5$ separable convolutions, $3 \times 3$ and $5 \times 5$ dilated separable convolutions, $3 \times 3$ max pooling, and $3 \times 3$ average pooling. All operations had a stride of one, and we zero-pad the input feature maps so that the size of the outputs did not change. Following $[8,9,11,15]$, we employed the ReLU-Conv-BN ordered sequence for convolution operations, and the separable convolutions were applied twice. Each cell contained $N=4$ nodes, each of which computed the output by concatenating the intermediate nodes in the channel dimension. The cells at $1 / 3$ and $2 / 3$ of the total depth of the network were reduction cells. In the reduction cells, all operations connected to the inputs of the cell had a stride of two. Therefore, there were two architecture parameters, $\alpha_{\text {normal }}$ and $\alpha_{\text {reduce }}$. These parameters were shared by each type of cell (e.g., $\alpha_{\text {normal }}$ was shared by all the normal cells) and were optimized by a stochastic gradient descent method through backpropagation.

\section{IMPLEMENTATION DETAILS}

\subsection{Training Settings}

The training settings were similar to those provided in [9]. In particular, we used the SGD with initial learning rate $\eta_{\alpha}=0.025$, momentum 0.9 , weight decay $3.0 \times 10^{-4}$, and mini-batch size of 128 . Throughout this study, the learning rate was annealed with cosine schedule [10]. We used the Adam [7] as the optimizer for the architecture parameters $\alpha$, with learning rate $\eta_{\alpha}=6.0 \times 10^{-4}$, momentum $\beta=(0.5,0.999)$, and weight decay $1.0 \times 10^{-3}$. Each continuous architecture was trained for 3 epochs, and the discrete architecture (i.e., the model with the most likely operations) was trained for 10 epochs.

After the architecture search was completed, a larger network of 20 cells with 36 initial channels were retrained for 600 epochs using all training data [9]. We followed the previous works [1,9,14], we additionally applied an auxiliary classifier [13] with a weight of 0.4, cutout [6] with length 16, ScheduleDropPath [15] with probability from 0 to 0.3 during the training. We then reported the test accuracy at the final epoch.

\subsection{Genetic Algorithm Settings}

We searched for the architectures using two strategies: evolutionary strategy (ES) [12] and non-dominated sorting genetic algorithms (NSGA-II) [5]. We first employed the ES framework to verify our network representation and extended it to multi-objective optimization using NSGA-II.

ES: For our ES configuration, we used $(1+2)$ ES with a mutation rate of 0.1 . We used only the uniform mutation for genetic operation. We chose the number of generations as $G=20$ and optimized the network architecture to maximize the fitness.

NSGA-II: For our NSGA-II configuration, we used the simulated binary crossover (SBX) [3] with probability $p=0.9$ and distribution index $\eta=3$, and polynomial mutation [2, 4] with distribution index $\eta=3$. We also applied weighted crossover with probability of 0.1 . We chose the number of generations as $G=15$ and population size as $N_{\text {pop }}=15$.

\subsection{Network architecture obtained by our method}

Figure 1 illustrates an example of networks (i.e., convolutional cell) with the lowest classification error and the lowest FLOPS picked from the final Pareto frontier. As can be seen in this figure, the network with the lowest FLOPS comprises several pooling operations in normal cell, which are relatively low latency ones.

\section{REFERENCES}

[1] Xin Chen, Lingxi Xie, Jun Wu, and Qi Tian. 2019. Progressive differentiable architecture search: Bridging the depth gap between search and evaluation. In CVPR. $1294-1303$.

[2] Kalyanmoy Deb. 2001. Multi-objective optimization using evolutionary algorithms. John Wiley \& Sons.

[3] Kalyanmoy Deb and Ram Bhushan Agrawal. 1995. Simulated binary crossover for continuous search space. Complex Systems 9 (1995), $115-148$.

[4] Kalyanmoy Deb and Samir Agrawal. 1999. A niched-penalty approach for constraint handling in genetic algorithms. In ANNGA. 235-243.

[5] Kalyanmoy Deb, Amrit Pratap, Sameer Agarwal, and Tanaka Meyarivan. 2002. A fast and elitist multiobjective genetic algorithm: NSGA-II. IEEE Transactions on Evolutionary Computation 6, 2 (2002), 182-197.

[6] Terrance DeVries and Graham W Taylor. 2017. Improved Regularization of Convolutional Neural Networks with Cutout. arXiv preprint:1805.09501 (2017).

[7] Diederik Kingma and Jimmy Ba. 2015. Adam: A method for stochastic optimization. In ICLR. 2452-2459. 


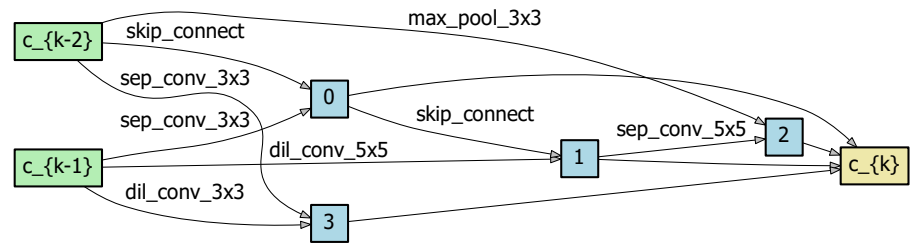

(a) Normal cell

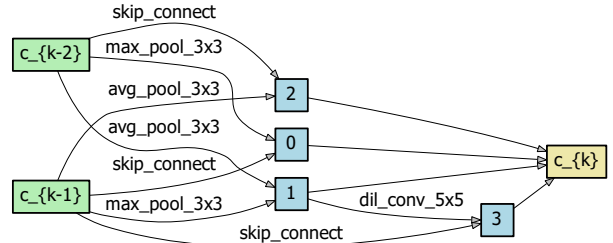

(b) Reduction cell

(a) The cells with lowest classification error.

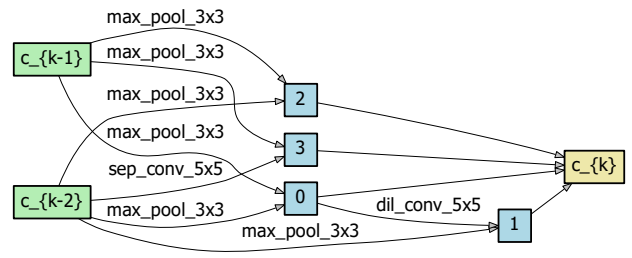

(a) Normal cell

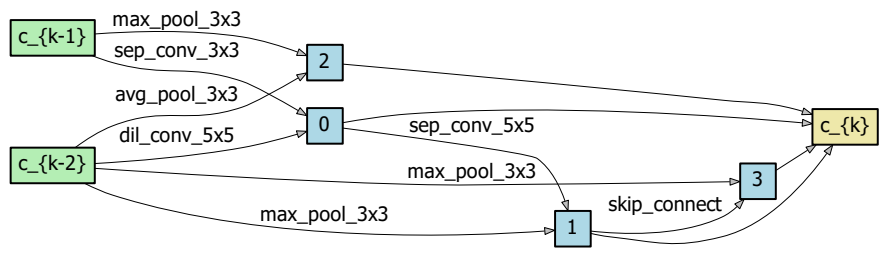

(b) Reduction cell

(b) The cells with lowest FLOPS.

Figure 1: One of the cells with lowest classification error (upper) and lowest FLOPS (lower).

[8] Chenxi Liu, Barret Zoph, Jonathon Shlens, Wei Hua, Li-Jia Li, Li Fei-Fei, Alan L. Yuille, Jonathan Huang, and Kevin Murphy. 2018. Progressive neural architecture search. In ECCV.

[9] Hanxiao Liu, Karen Simonyan, and Yiming Yang. 2019. DARTS: Differentiable Architecture Search. In ICLR.

[10] Ilya Loshchilov and Frank Hutter. 2017. SGDR: Stochastic Gradient Descent with Restarts. In ICLR

[11] Esteban Real, Alok Aggarwal, Yanping Huang, and Quoc V. Le. 2019. Regularized evolution for image classifier architecture search. In AAAI. 4780-4789.

[12] Ingo Rechenberg. 1978. Evolutionsstrategien. Simulationsmethoden in der Medizin und Biologie (1978), 83-114.

[13] Christian Szegedy, Vincent Vanhoucke, Sergey Ioffe, Jonathon Shlens, and Zbigniew Wojna. 2016. Rethinking the Inception Architecture for computer vision. In ICCV. $2818-2826$.

[14] Sirui Xie, Hehui Zheng, Chunxiao Liu, and Liang Lin. 2019. SNAS: stochastic neural architecture search. In ICLR.

[15] Barret Zoph, Vijay Vasudevan, Jonathon Shlens, and Quoc V. Le. 2018. Learning transferable architectures for scalable image recognition. In CVPR. 8697-8710. 